\title{
A New Application of Human Visual Simulated Images in Optometry Services
}

\author{
Lin-Song Chang and Bo-Wen Wu* \\ Department Optometry, College of Medical Technology, Nursing and Wellbeing, Yuanpei University, \\ No. 306, Yuanpei St., Hsinchu 30015, Taiwan, R.O.C.
}

(Received May 21, 2013 : revised July 19, 2013 : accepted July 22, 2013)

\begin{abstract}
Due to the rapid advancement of auto-refractor technology, most optometry shops provide refraction services. Despite their speed and convenience, the measurement values provided by auto-refractors include a significant degree of error due to psychological and physical factors. Therefore, there is a need for repetitive testing to obtain a smaller mean error value. However, even repetitive testing itself might not be sufficient to ensure accurate measurements. Therefore, research on a method of measurement that can complement auto-refractor measurements and provide confirmation of refraction results needs to be conducted. The customized optometry model described herein can satisfy the above requirements. With existing technologies, using human eye measurement devices to obtain relevant individual optical feature parameters is no longer difficult, and these parameters allow us to construct an optometry model for individual eyeballs. They also allow us to compute visual images produced from the optometry model using the CODE $\mathrm{V}$ macro programming language before recognizing the diffraction effects visual images with the neural network algorithm to obtain the accurate refractive diopter. This study attempts to combine the optometry model with the back-propagation neural network and achieve a double check recognition effect by complementing the auto-refractor. Results show that the accuracy achieved was above $98 \%$ and that this application could significantly enhance the service quality of refraction.
\end{abstract}

Keywords : Optometry model, Simulating ametropia, Auto-refractor, BPNN recognition, Invariant moments OCIS codes : (200.0200) Optics in computing; (330.4875) Optics of physiological systems; (330.7327) Visual optics, ophthalmic instrumentation; (330.5000) Vision - patterns and recognition

\section{INTRODUCTION}

Due to advancements in living standards and refraction technology, refraction itself is no longer purely an ametropia problem. There is now a growing desire to construct an individual unique optometry model and integrate refraction as part of the test of visual quality. Saving time and customizing designs of refraction are now popular research, as well as application, topics.

The optical principle of refraction entails locating the conjugate focus of the retina in the focusing process. The refractive diopter can then be computed from the conjugate focus distance. Most research in this field focuses on measurement technique and the accuracy of auto-refractors or discusses subjective refraction and objective refraction [1-3]. Few studies, however, touch on the above visual technique topics from the viewpoint of the optometry model, to say nothing of using the optometry model to complement refraction services [4].

Segmenting the optical system of the eye into the cornea, aqueous, crystalline lens, vitreous, retina, and other systems and determining the sub-system parameters would allow us to use CODE V optics design software to add in emmetropia, the sub-system of a set of lens simulations known as ametropia. This would result in a new set of optical systems for the eye that would have its own refractive diopter. There would be both collective and individual factors to consider in the aforementioned process, but fortunately, the characteristics of $\mathrm{CODE} \mathrm{V}$ macro programming language satisfy these requirements.

Chapter 1 of this study describes the principle and construction of the optometry model. Chapter 2 simulates the visual image produced. Chapter 3 describes the back propagation neural network (BPNN) principle. Chapter 4 describes the

\footnotetext{
*Corresponding author: wubwen@mail.ypu.edu.tw

Color versions of one or more of the figures in this paper are available online.
} 
recognition method using invariant moments as the feature value. Chapter 5 discusses the experimental results. Finally we state the conclusions.

\section{METHODS}

\subsection{Construction of a Model for the Human Optical System}

Ever since Gullstrand first proposed the optometry model in 1911, many researchers have proposed improved versions of the model [5] that would increase the eye aspheric surface and change the refractive index of crystalline lens to a variation value. The most complete and realistic proposal was the eye optometry model mentioned by Liou in 1997 [6]. The cornea and crystalline lens in the model adopted the aspheric coefficient and used a varying refractive index with relevant parameters, as shown in Table 1. The computation formula for the model's refractive index is shown in equations (1)-(3):

$$
\begin{aligned}
\operatorname{Grad} \mathrm{A} & =n(r, z)=n_{0}+\left(N_{r 2}\right) z+\left(N_{z 1}\right) z^{2}+\left(N_{z 2}\right) r^{2} \\
& =1.368+0.049057 . \mathrm{z}^{-0.015427} \cdot \mathrm{z}^{2}-0.001978 \times \mathrm{r}^{2}
\end{aligned}
$$

The distribution of the gradient index is represented in the form.

' $z$ ' is along the optical axis, ' $r$ ' is the radial distance perpendicular to the $\mathrm{z}$ axis $\left(\mathrm{r}^{2}=\mathrm{x}^{2}+\mathrm{y}^{2}\right)$.
Grad $P=1.407-0.006605 . \quad z^{2}-0.001978 . \quad r^{2}$

The different ocular refractive indices at various wavelengths $\lambda$ can be found as

$$
\mathrm{n}(\lambda)=\mathrm{n}(\text { media at } 0.555 \mu \mathrm{m})+0.0512-0.1455 . \quad \lambda+0.0961 . \quad \lambda^{2}
$$

This study used the Liou optometry model as a basis for constructing an original optometry model. This original model was then used to simulate the actual degree of human eye ametropia, before conducting visual image simulation by inserting macro language into CODE $\mathrm{V}$.

The Liou eye model built using CODE V [7] is shown in Fig. 1. The various optic parameters of the optometry model are shown in Table 2.

\subsection{Simulating Ametropia in the Optometry Model}

In order to simulate the ametropia of the human eye, two surfaces (one lens) were added to the corneal vertex of the original optometry model. The sphere lens (radius of curvature of 200 millimeters and -200 millimeters) simulated a myopic eyesight of $5.00 \mathrm{D}$, as shown in Fig. 2. The various optic parameters of ametropia in the optometry model are shown in Table 3.

From the ametropic eye, as shown in Fig. 2, it can be seen that due to the existence of myopia, the focus on the retina becomes blurred. Therefore, the visual image produced

TABLE 1. Relevant parameters of the Liou optometry model

\begin{tabular}{c|c|c|c|c}
\hline \multicolumn{5}{c}{ Liou optometry model (1997) } \\
\hline Surface & Radius ( mm) & Thickness ( mm) & Asphericity coefficient & Refraction index (555 $\mathrm{nm})$ \\
\hline 1 & 7.77 & 0.50 & -0.18 & 1.376 \\
\hline 2 & 6.4 & 3.16 & -0.60 & 1.336 \\
\hline 3 & 12.4 & 1.59 & -0.94 & Grad A \\
\hline 4 & Infinity & 2.43 & - & Grad P \\
\hline 5 & -8.10 & 16.27 & 0.96 & 1.336 \\
\hline
\end{tabular}

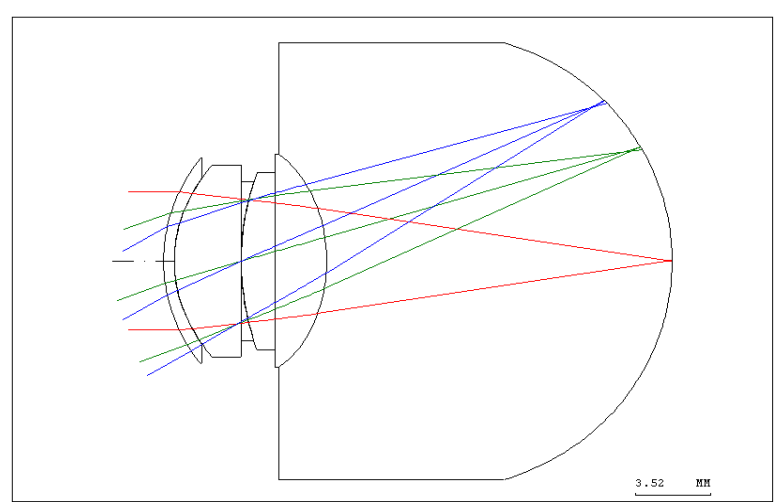

FIG. 1. An original optometry model.
TABLE 2. The various optic parameters of the original optometry model

\begin{tabular}{c|c|c|c|c|c|c}
\hline \hline $\begin{array}{c}\text { Surface } \\
\#\end{array}$ & $\begin{array}{c}\text { Surface } \\
\text { Type }\end{array}$ & Y Radius & Thickness & Glass & Refract & $\begin{array}{c}\text { Y Semi- } \\
\text { Aperture }\end{array}$ \\
\hline Object & Sphere & infinity & infinity & & Refract & \\
\hline 1 & Conic & 7.7700 & 0.5000 & 'oomea' & Refract & 4.3389 \\
\hline 2 & Conic & 6.4000 & 3.1600 & 'humor' & Refract & 4.0280 \\
\hline Stop & Sphere & infinity & 0.0000 & 'humor' & Refract & 3.0000 \\
\hline 4 & Conic & 12.4000 & 1.5900 & 'Grad A' & Refract & 2.9688 \\
\hline 5 & Sphere & infinity & 2.4300 & 'Grad P' & Refract & 3.2961 \\
\hline 6 & Conic & -8.1000 & 16.2700 & 'humor' & Refract & 3.7167 \\
\hline Image & Sphere & -11.0000 & 0.0000 & & Refract & 9.6201 \\
\hline
\end{tabular}




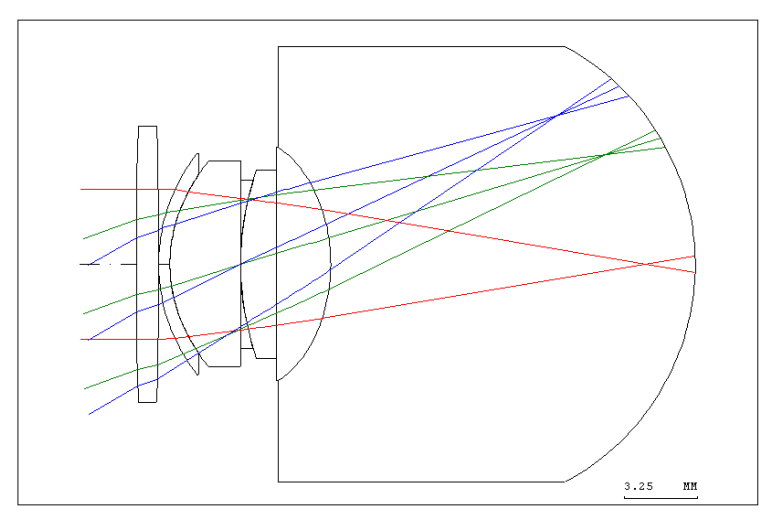

FIG. 2. The optometry model simulating ametropia in diopters.

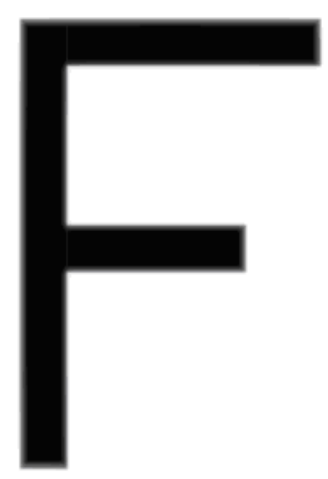

(a)

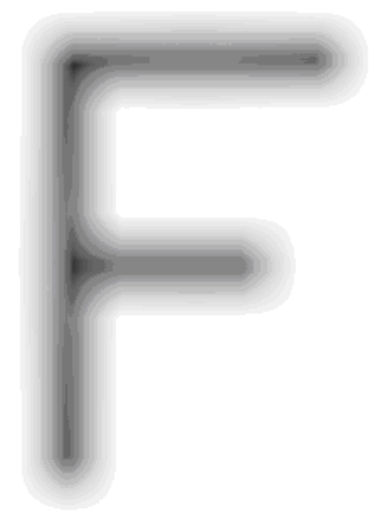

(b)
FIG. 3. Simulated human visual images, (a) visual image of emmetropia (b) visual image of ametropia in diopters.

by the human eye was also blurred due to ametropia. However, the visual images contain diffraction effects, so the location of the center of the image is relatively clear. The visual image of emmetropia is shown in Fig. 3(a), whereas the visual image of ametropia in diopters is shown in Fig. 3(b).

\subsection{Back Propagation Neural Network (BPNN)}

The learning process of a BPNN comprises feed-forward signal propagation and feedback signal propagation. In the process of feed-forward signal propagation, the input information is routed through hidden levels for weighting operation and, after going through transformation by Activation Function, is redirected to output level for output value operation. The neural elements in each level only affect the status at the neural elements of the next level. The operation switches to the feedback signal propagation when the output level does not meet the target value. The difference signals are fed back along the original paths to correct the weighting and bias of the neural elements at all levels. The operation
TABLE 3. Various optic parameters of the optometry model simulating ametropia in diopters

\begin{tabular}{c|c|c|c|c|c|c}
\hline $\begin{array}{c}\text { Surface } \\
\#\end{array}$ & $\begin{array}{c}\text { Surface } \\
\text { Type }\end{array}$ & Y Radius & Thickness & Glass & Refract & $\begin{array}{c}\text { Y Semi- } \\
\text { Aperture }\end{array}$ \\
\hline Object & Sphere & infinity & infinity & & Refract & \\
\hline 1 & Sphere & 200.00000 & 1.00000 & & Refract & 5.44252 \\
\hline 2 & Conic & -200.00000 & 0.00000 & & Refract & 5.15306 \\
\hline 3 & Conic & 7.77000 & 0.50000 & 'oomea' & Refract & 4.30030 \\
\hline 4 & Conic & 6.40000 & 3.16000 & 'humor' & Refract & 3.97539 \\
\hline Stop & Sphere & infinity & 0.0000 & 'humor' & Refract & 3.0000 \\
\hline 6 & Conic & 12.4000 & 1.59000 & 'Grad A' & Refract & 2.89414 \\
\hline 7 & Sphere & infinity & 2.4300 & 'Grad P' & Refract & 3.20049 \\
\hline 8 & Conic & -8.1000 & 16.2700 & 'humor' & Refract & 3.63106 \\
\hline Image & Sphere & -11.0000 & 0.0000 & & Refract & 8.80468 \\
\hline & & & & & \multicolumn{1}{|c}{}
\end{tabular}

stops when the differences are within the acceptable error ranges.

In the BPNN, the input of neural element $\mathrm{j}$ at level $n$ denotes the non-linear function of the output of the neural elements at level n-1.

$\mathrm{y}_{\mathrm{k}}^{\mathrm{n}}$ : output of the $k$ th neural element at output of $n$th level

$\mathrm{f}:$ Activation Function

net $_{\mathrm{j}}^{\mathrm{n}}$ : sum of weighting of the output at $n-1$ th level

$\mathrm{w}_{\mathrm{ji}}^{\mathrm{n}}$ : linking weighting of the neural elements between $j$ th

element at $n$th level and $i$ th element at $n-1$ th level

$\mathrm{b}_{\mathrm{j}}^{\mathrm{n}}$ : bias of the $j$ th neural element at $n$th level

$\mathrm{E}$ : error function

$\mathrm{d}_{\mathrm{k}}$ : target output value of the $k$ th neural element

$\rho$ : learning rate, determining the correction scale of the gradient steepest descent method

$$
\begin{aligned}
& y_{i}^{n}=f\left(\operatorname{net}_{j}^{n}\right), \quad \operatorname{net}_{j}^{n}=\sum_{i} w_{j i}^{n} y_{i}^{n-1}-b_{j}^{n} \\
& E=(1 / 2) \sum_{k}\left(d_{k}-y_{k}\right)^{2}, \quad w_{j i}=-\rho \frac{\partial E}{\partial w_{j i}}
\end{aligned}
$$

Due to the supervised learning nature of the BPNN, with the aim of reducing the difference between output and target values, the learning process of the network is to minimize the error function ' $E$ '. This study adopts the gradient steepest descent method to search the optimum solution on ' $E$ ' (the least squares of tolerances). Upon receiving an input data, the network adjusts the weighting values in proportion to the extent of sensitivity of the error function to weighting. Optimum recognition results can be achieved, through repeated computation and learning, once a minimum ' $\mathrm{E}$ ' is found or a state of convergence is reached [8].

The program sequence of BPNN thermal image recognition 
is as follows:

Step 1: Determine the numbers of network levels and neural elements between layers.

Step 2: Set the initial weighting and bias of the network to a random number.

Step 3: Input training samples and target outputs.

Step 4: Calculate the sum of squares of tolerances between the values at the output level and the target output values.

Step 5: Calculate the difference between the output and hidden layers.

Step 6: Calculate the corrections of weighting and bias between layers.

Step 7: Update the weighting and bias between layers.

Step 8: Repeat steps 3-7 until convergence, or until the minimum is reached.

Step 9: Perform the BPNN test.

Step 9-1: Set the numbers of layers and neural elements between layers

Step 9-2: Read the weighting and bias going through training.

Step 9-3: Input a test example.

Step 9-4: Calculate the output.

Step 10: Recall unknown or un-classified images with the accepted neural network.

\section{RESULTS}

\subsection{Recognition of the Visual Image of Human Eye Ametropia}

A total of 24 optometry models were created for eye models with myopia of diopter $(-0.25 \mathrm{D} \sim-6.00 \mathrm{D})$, and each model was increased by $-0.25 \mathrm{D}$. These would lead to corresponding visual images, with each visual image resulting in 4 visual images with different object field angles $\left(1.5^{\circ}\right.$, $2^{\circ}, 2.5^{\circ}, 3^{\circ}$ ) as shown in Fig. 4. The resulting visual images have different angles, though the corresponding refractive diopters are similar.

Each ametropia has 4 different direction images, which can be divided into 24 categories (numbers 1 24). Therefore, a total of 96 images were utilized, of which 48 images were provided for the BPNN for training and learning purposes, and the remaining 48 images were utilized for testing and verification purposes. Additionally, the obtained diopter ("D") were adopted as the output of the BPNN.

These images' effective features were obtained as inputs to the BPNN. The BPNN was then trained to recognize objects based on the features. This study adopts the theorem of invariant moments (Hu's moments) [9], by which normalized moments of the given image's absolute value were taken as features, as shown in Table 4.

Some basic geometric features in a two-dimensional domain include magnitude, position, direction and shape, and most bear some relationship to moment.

$$
\begin{aligned}
& \bar{x}, \bar{y}: \text { the centroid of the image } \\
& \mathrm{m}_{00}: \text { the pixel is equal to } 1 \text { of total } \\
& \mathrm{m}_{10}: \text { the moment of the ' } \mathrm{x} \text { ' direction } \\
& \mathrm{m}_{01} \text { : the moment of the 'y' direction }
\end{aligned}
$$

According to the invariant moments proposed by Hu's [9], we can present the gray level value in the figure using the two-dimensional distribution matrix of $\mathrm{b}(\mathrm{m}, \mathrm{n})$; its $(p+q)$ order moment of the image distribution is expressed below:

$$
\mathrm{m}_{\mathrm{pq}}=\sum_{\mathrm{m}=0}^{\mathrm{m}-1} \sum_{\mathrm{n}=0}^{\mathrm{n}-1} \mathrm{~m}^{\mathrm{p}} \mathrm{n}^{\mathrm{q}} \mathrm{b}(\mathrm{m}, \mathrm{n}) \quad \mathrm{p}, \mathrm{q}=0,1,2, \ldots
$$

The central moment (Eq. (6)), which has the property of invariance to translation, is shown below.

$$
\begin{aligned}
& \mu_{\mathrm{pq}}=\sum_{\mathrm{m}=0}^{\mathrm{m}-1} \sum_{\mathrm{n}=0}^{\mathrm{n}-1}(\mathrm{~m}-\overline{\mathrm{x}})^{\mathrm{p}}(\mathrm{n}-\overline{\mathrm{y}})^{\mathrm{q}} \mathrm{b}(\mathrm{m}, \mathrm{n}) \quad \overline{\mathrm{x}}=\frac{\mathrm{m}_{10}}{\mathrm{~m}_{00}}, \quad \overline{\mathrm{y}}=\frac{\mathrm{m}_{01}}{\mathrm{~m}_{00}} \\
& \eta_{\mathrm{pq}}=\frac{\mu_{\mathrm{p}, \mathrm{q}}}{\mu_{00}^{(\mathrm{p}+\mathrm{q}+2) / 2}} \quad \text { (Normalization of the central moment) }
\end{aligned}
$$

$\mathrm{Hu}$ derived the following seven invariant functions[10]. The seven terms listed below (Eq. (7)) were adopted in the following training and test set, as inputs to the network, instead of the actual pixel gray levels.
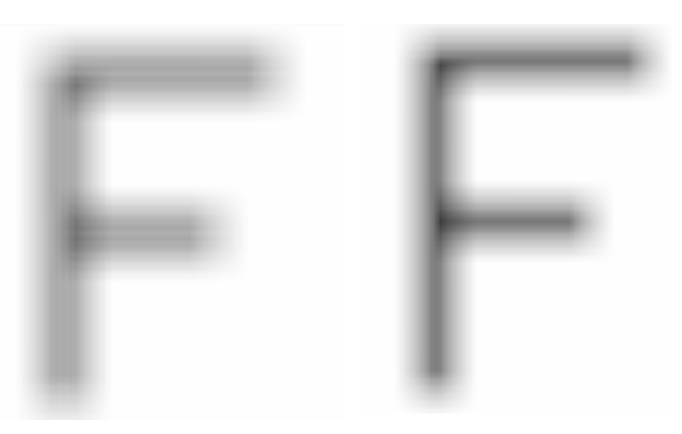
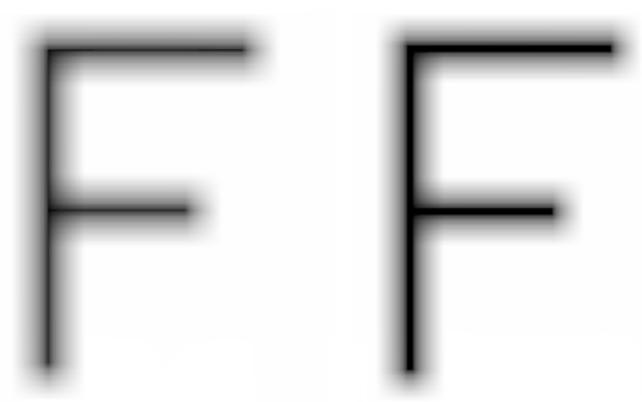

FIG. 4. Four parts of image files. 
TABLE 4. Normalization of invariant moments of the 24 eye optometry model visual image files

\begin{tabular}{c|c|c|c|c|c|c|c}
\hline \hline Sample/Invariants & $\Phi 1$ & $\Phi 2$ & $\Phi 3$ & $\Phi 4$ & $\Phi 5$ & $\Phi 6$ & $\Phi 7$ \\
\hline Diopter 1 & 0.1208 & 0.3792 & 0.5044 & 0.4670 & 0.9917 & 0.6697 & 0.9476 \\
\hline Diopter 2 & 0.1208 & 0.3843 & 0.5044 & 0.4669 & 0.9905 & 0.6716 & 0.9474 \\
\hline Diopter 3 & 0.1207 & 0.3868 & 0.5049 & 0.4671 & 0.9900 & 0.6725 & 0.9478 \\
\hline Diopter 4 & 0.1207 & 0.3776 & 0.5049 & 0.4673 & 0.9896 & 0.6692 & 0.9484 \\
\hline Diopter 5 & 0.1208 & 0.3776 & 0.5036 & 0.4669 & 0.9874 & 0.6693 & 0.9471 \\
\hline Diopter 6 & 0.1207 & 0.3808 & 0.5043 & 0.4668 & 0.9940 & 0.6695 & 0.9473 \\
\hline Diopter 7 & 0.1207 & 0.3787 & 0.5046 & 0.4670 & 0.9941 & 0.6688 & 0.9477 \\
\hline Diopter 8 & 0.1208 & 0.3814 & 0.5036 & 0.4666 & 0.9861 & 0.6704 & 0.9467 \\
\hline Diopter 9 & 0.1207 & 0.3838 & 0.5046 & 0.4666 & 0.9915 & 0.6703 & 0.9472 \\
\hline Diopter 10 & 0.1207 & 0.3812 & 0.5047 & 0.4668 & 0.9927 & 0.6696 & 0.9475 \\
\hline Diopter 11 & 0.1208 & 0.3916 & 0.5053 & 0.4669 & 0.9917 & 0.6734 & 0.9478 \\
\hline Diopter 12 & 0.1208 & 0.3840 & 0.5048 & 0.4668 & 0.9968 & 0.6704 & 0.9475 \\
\hline Diopter 13 & 0.1208 & 0.3793 & 0.5040 & 0.4668 & 0.9870 & 0.6697 & 0.9471 \\
\hline Diopter 14 & 0.1208 & 0.3837 & 0.5050 & 0.4669 & 0.9950 & 0.6704 & 0.9477 \\
\hline Diopter 15 & 0.1208 & 0.3852 & 0.5046 & 0.4670 & 0.9903 & 0.6717 & 0.9475 \\
\hline Diopter 16 & 0.1207 & 0.3848 & 0.5045 & 0.4669 & 0.9903 & 0.6713 & 0.9474 \\
\hline Diopter 17 & 0.1208 & 0.3789 & 0.5039 & 0.4666 & 0.9920 & 0.6687 & 0.9470 \\
\hline Diopter 18 & 0.1208 & 0.3811 & 0.5047 & 0.4669 & 0.9935 & 0.6700 & 0.9476 \\
\hline Diopter 19 & 0.1208 & 0.3869 & 0.5052 & 0.4669 & 1.0000 & 0.6712 & 0.9477 \\
\hline Diopter 20 & 0.1208 & 0.3810 & 0.5032 & 0.4666 & 0.9836 & 0.6707 & 0.9465 \\
\hline Diopter 21 & 0.1207 & 0.3855 & 0.5047 & 0.4666 & 0.9891 & 0.6715 & 0.9472 \\
\hline Diopter 22 & 0.1208 & 0.3791 & 0.5040 & 0.4667 & 0.9909 & 0.6689 & 0.9470 \\
\hline Diopter 23 & 0.1208 & 0.3857 & 0.5055 & 0.4670 & 0.9943 & 0.6716 & 0.9481 \\
\hline Diopter 24 & 0.1207 & 0.3981 & 0.5056 & 0.4671 & 0.9904 & 0.6764 & 0.9481 \\
\hline
\end{tabular}

$$
\begin{aligned}
& \phi_{1}=\eta_{20}+\eta_{02} \\
& \phi_{2}=\left(\eta_{20}-\eta_{02}\right)^{2}+4 \eta_{11}^{2} \\
& \phi_{3}=\left(\eta_{30}-3 \eta_{12}\right)^{2}+\left(\eta_{03}-3 \eta_{21}\right)^{2} \\
& \phi_{4}=\left(\eta_{30}+\eta_{12}\right)^{2}+\left(\eta_{03}+\eta_{21}\right)^{2} \\
& \phi_{5}=\left(3 \eta_{30}-3 \eta_{12}\right)\left(\eta_{30}+\eta_{12}\right)\left[\left(\eta_{30}+\eta_{12}\right)^{2}-3\left(\eta_{21}+\eta_{03}\right)^{2}\right] \\
& +\left(3 \eta_{21}-\eta_{03}\right)\left(\eta_{21}+\eta_{03}\right) \times\left[3\left(\eta_{30}+\eta_{12}\right)^{2}-\left(\eta_{21}+\eta_{03}\right)^{2}\right] \\
& \phi_{6}=\left(\eta_{20}-\eta_{02}\right)\left[\left(\eta_{30}+\eta_{12}\right)^{2}-\left(\eta_{21}+\eta_{03}\right)^{2}\right]+4 \eta_{11}\left(\eta_{30}+\eta_{12}\right)\left(\eta_{21}+\eta_{03}\right) \\
& \phi_{7}=\left(3 \eta_{21}-\eta_{03}\right)\left(\eta_{30}+\eta_{12}\right)\left[\left(\eta_{30}+\eta_{12}\right)^{2}-3\left(\eta_{21}+\eta_{03}\right)^{2}\right] \\
& +\left(3 \eta_{12}-\eta_{30}\right)\left(\eta_{21}+\eta_{03}\right) \times\left[3\left(\eta_{30}+\eta_{12}\right)^{2}-\left(\eta_{21}+\eta_{03}\right)^{2}\right]
\end{aligned}
$$

\section{DISCUSSION}

\subsection{Discussion of Experimental Results}

A total of 96 imaging samples at different ametropia were recognized and sorted by the BPNN. Table 5 lists the BPNN parameters associated with the 24 elements.
TABLE 5. Network parameters settings

\begin{tabular}{c|c}
\hline \hline Input unit & 7 units $(\Phi 1, \Phi 2, \Phi 3, \Phi 4, \Phi 5, \Phi 6, \Phi 7)$ \\
\hline Hide unit & 16 units \\
\hline Out unit & 24 units (diopters) \\
\hline The number of time & 50000 times \\
\hline Learning rate & 0.5 \\
\hline Momentum term & 0.5 \\
\hline Training and test sample & $\begin{array}{c}48 \text { training samples, 48 testing and } \\
\text { verification samples }\end{array}$ \\
\hline
\end{tabular}

Table 6 shows the weighting and bias with convergence. Fig. 5 displays the training curve. Table 7 presents the recognition results. Results show that the accuracy achieved was above 98\%, as shown in Fig. 6.

\section{CONCLUSION}

The biggest contribution of this paper was the develop- 
TABLE 6. Diopter weighting and bias with network convergence

\begin{tabular}{|c|c|c|c|c|c|c|c|c|c|c|c|c|c|c|c|c|}
\hline \multirow{16}{*}{$\begin{array}{l}\text { Weighting: } \\
\text { [Input][Hide] } \\
=[7][16]\end{array}$} & \multicolumn{2}{|c|}{-2.65} & \multicolumn{3}{|c|}{75.29} & \multicolumn{2}{|c|}{-5.80} & \multicolumn{2}{|c|}{-7.88} & \multicolumn{3}{|c|}{-10.29} & \multicolumn{2}{|l|}{20.35} & \multicolumn{2}{|c|}{-10.97} \\
\hline & \multicolumn{2}{|c|}{-0.46} & \multicolumn{3}{|c|}{20.68} & \multicolumn{2}{|c|}{-0.27} & \multicolumn{2}{|c|}{-1.37} & \multicolumn{2}{|r|}{-7.62} & & \multicolumn{2}{|l|}{6.28} & \multicolumn{2}{|c|}{-2.70} \\
\hline & \multicolumn{2}{|c|}{2.31} & \multicolumn{3}{|c|}{-66.29} & \multicolumn{2}{|c|}{1.54} & \multicolumn{2}{|c|}{6.27} & & 5.88 & & -15.04 & & 9.9 & \\
\hline & & .03 & & -0.48 & & -1.00 & & -0 . & 54 & & -1.55 & & -0.72 & & -1.8 & \\
\hline & & .53 & & 89.28 & & -8.65 & & -14 & .55 & & 22.03 & & 12.63 & & -19.8 & \\
\hline & & .37 & & 9.95 & & 0.15 & & -0 . & 31 & & -6.27 & & 3.30 & & -1.5 & \\
\hline & & .25 & & 59.90 & & -5.0 & & -6 . & 85 & & -4.13 & & 14.88 & & -9.6 & \\
\hline & & .43 & & -108.92 & & 4.71 & & 13 & 32 & & -4.21 & & -20.57 & & 19.2 & \\
\hline & & .11 & & 2.13 & & -0.56 & & -0 . & 32 & & -2.76 & & 0.22 & & -1.0 & \\
\hline & & .93 & & 94.93 & & -9.12 & & -12 & 26 & & 3.59 & & 21.00 & & -16 . & \\
\hline & & .01 & & -0.93 & & -0.8 & & -0 . & 76 & & -1.26 & & -1.23 & & -1.6 & \\
\hline & & .61 & & -96.62 & & 9.05 & & 13 & 94 & & -0.99 & & -18.36 & & 19.4 & \\
\hline & & .75 & & 49.23 & & -3.6 & & -5 . & 69 & & -4.12 & & 11.82 & & -7.9 & \\
\hline & & .03 & & 40.08 & & -2.23 & & -3 & 21 & & -10.76 & & 11.66 & & -5.0 & \\
\hline & & .13 & & 70.51 & & -6.5 & & -9 & 33 & & 2.42 & & 15.46 & & -12. & \\
\hline & & .14 & & 1.32 & & -0.31 & & -0 . & 58 & & -2.73 & & 0.18 & & -0.8 & \\
\hline & -2.46 & -0.07 & -2.76 & -0.84 & -2.47 & -0.50 & -1.30 & -3.43 & -0.58 & -6.47 & -1.68 & 1.04 & 1.35 & 0.28 & -4.36 & -0.94 \\
\hline & -1.05 & 0.01 & -2.09 & -0.67 & 0.61 & -1.23 & 0.10 & -4.63 & -1.04 & -2.43 & -1.42 & -1.62 & 0.61 & -0.19 & -1.47 & -1.33 \\
\hline & -2.31 & -1.70 & -5.87 & -0.61 & 0.02 & -2.29 & -1.55 & -13.8 & -0.77 & -2.14 & -1.29 & -2.52 & -2.7 & -2.61 & -1.60 & -1.35 \\
\hline & -6.38 & -2.12 & 0.65 & -0.81 & -5.62 & -1.32 & -4.83 & -1.21 & -0.37 & -9.86 & -0.77 & 1.90 & -3.28 & -2.38 & -7.59 & -0.18 \\
\hline & \begin{tabular}{|l|}
-7.38 \\
\end{tabular} & -2.17 & 3.00 & -0.17 & -10.38 & -0.65 & -6.34 & -0.13 & 0.19 & -12.29 & -0.72 & 2.86 & -4.64 & -3.06 & -8.40 & 0.15 \\
\hline & -3.86 & -1.46 & -2.86 & -0.55 & -1.78 & -0.93 & -2.35 & -3.21 & -0.59 & -3.47 & -0.56 & 0.53 & -1.44 & -1.91 & -1.78 & -0.75 \\
\hline & -9.97 & -3.97 & 1.20 & -0.22 & -4.47 & -1.12 & -6.96 & -0.14 & -0.2 & -12.47 & -0.67 & 2.62 & -5.09 & -5.85 & -7.88 & -0.28 \\
\hline & -9.75 & -0.56 & -1.11 & -0.47 & -10.28 & 0.16 & -8.04 & -1.40 & -0.15 & -17.50 & -0.53 & 1.16 & -5.97 & -2.61 & -11.63 & -0.43 \\
\hline & -4.45 & 0.17 & -4.08 & -0.43 & -2.04 & 0.04 & -3.25 & -4.55 & -0.09 & -6.44 & -0.60 & -0.64 & -2.77 & -1.08 & -4.11 & -0.84 \\
\hline & -5.91 & 0.25 & 1.73 & -0.20 & -5.47 & 0.21 & -4.49 & 0.85 & -0.73 & -11.03 & -0.41 & 0.75 & -3.36 & -0.37 & -6.85 & -0.86 \\
\hline & -2.08 & -1.81 & -2.18 & -0.70 & -0.13 & -2.18 & -1.28 & -7.62 & -0.98 & 1.45 & -0.65 & -6.97 & -3.62 & -3.9 & 0.45 & -1.36 \\
\hline rHids & -4.62 & -0.63 & -5.02 & -0.23 & 0.09 & -1.04 & -2.94 & -8.73 & -0.48 & -4.12 & -0.55 & -3.06 & -2.31 & -1.63 & -2.23 & -0.98 \\
\hline $\begin{array}{c}=[16][24] \\
=\end{array}$ & -7.62 & -1.59 & 2.3 & -0.05 & -7.36 & 0.30 & -5.92 & 1.39 & -0.02 & -10.8 & -0.81 & -1.14 & -4.27 & -2.53 & -7.35 & -0.20 \\
\hline & -3.45 & -0.60 & -1.48 & -0.49 & -0.47 & -0.53 & -2.05 & -3.53 & -0.78 & -3.48 & -0.85 & -2.71 & -1.42 & -1.04 & -2.14 & -1.00 \\
\hline & -3.21 & -0.27 & -2.40 & -0.4 & -0.62 & -0.38 & \begin{tabular}{|l|}
-1.89 \\
\end{tabular} & -4.74 & \begin{tabular}{|l|}
-0.37 \\
\end{tabular} & -2.11 & -0.36 & -2.78 & -1.87 & \begin{tabular}{|l|}
-1.09 \\
\end{tabular} & -1.56 & -0.60 \\
\hline & -3.93 & -0.01 & -5.30 & -0.28 & -1.22 & -0.70 & -2.62 & -9.27 & -0.34 & -4.25 & -0.51 & -4.12 & -2.18 & \begin{tabular}{|l|}
-0.99 \\
\end{tabular} & -2.43 & -0.55 \\
\hline & -9.50 & -2.07 & 3.50 & -0.47 & -11.16 & -0.63 & -7.25 & 2.14 & -0.39 & -12.1 & -0.61 & -2.1 & -5.93 & -4.20 & -9.42 & -0.46 \\
\hline & \begin{tabular}{|l|}
-4.58 \\
\end{tabular} & -1.29 & 3.62 & -0.43 & \begin{tabular}{|l|}
-7.53 \\
\end{tabular} & -0.36 & -3.43 & 4.09 & \begin{tabular}{|l|}
-0.49 \\
\end{tabular} & -7.54 & -0.77 & -3.24 & -2.33 & -2.60 & -5.87 & -0.41 \\
\hline & -5.68 & -1.27 & -4.69 & -0.21 & 1.02 & -1.23 & -3.39 & -9.98 & -0.84 & -2.12 & -0.58 & -5.61 & -2.85 & -2.78 & -1.17 & -1.06 \\
\hline & -3.15 & -0.18 & -2.28 & -0.58 & -2.77 & 0.01 & -2.06 & -2.23 & -0.57 & -5.29 & -0.46 & -3.09 & -0.48 & -0.10 & -3.12 & -0.44 \\
\hline & -4.29 & -0.43 & -5.71 & -0.28 & -0.33 & -1.16 & -2.87 & \begin{tabular}{|l|}
-7.99 \\
\end{tabular} & -0.53 & -2.1 & -0.63 & -2.23 & -2.39 & -1.71 & -0.93 & -0.84 \\
\hline & -6.75 & -1.95 & 0.26 & -0.2 & -4.06 & -1.14 & -4.98 & -0.16 & -0.04 & -8.51 & -0.15 & 2.37 & -2.05 & -2.53 & -5.69 & 0.21 \\
\hline & -8.50 & -0.48 & -11.85 & \begin{tabular}{|l|l|}
5 & -0.35 \\
\end{tabular} & 0.38 & -0.85 & -4.73 & -17.05 & 0.46 & -5.79 & -0.78 & 1.41 & -2.71 & -2.72 & -2.38 & 0.20 \\
\hline & 7.56 & 4.59 & -14.55 & \begin{tabular}{|l|l|}
5 \\
\end{tabular} & 6.79 & 2.10 & 7.13 & -17.17 & 1.82 & 6.35 & -0.38 & -24.11 & 6.87 & 6.01 & 6.85 & 1.45 \\
\hline Bias hide & 17.89 & 3.66 & -14.32 & 21.94 & 34.66 & 1.58 & 16.33 & -30.64 & 1.23 & 28.91 & 2.23 & -30.56 & 13.28 & 7.64 & 21.88 & 1.20 \\
\hline & 1.91 & 0. & 37 & -3.30 & 1.24 & 2. & 21 & 1.07 & 1.67 & & .11 & -0.74 & 1.43 & & .60 & -3.54 \\
\hline $\mathrm{D}$ & -1.36 & -1. & .81 & -1.98 & -5.27 & -3 & .78 & -2.86 & -4.66 & & .02 & -1.81 & 2.82 & & .39 & 4.45 \\
\hline
\end{tabular}

ment of a method that could complement the auto-refractor, taking advantage of its convenience and speed while avoiding its instability. The BPNN recognition method used could flexibly adjust the number of hide units, enhancing the speed of curve convergence through the neural network algorithm and recall capability. Together with invariant moments independent of visual images and revolving characteristics, this method could enhance the recognition of visual images to $98 \%$, achieving results through subjective scientific methods not possible from the human eye.
Using the relevant parameters of the Liou model as a theoretical model and using the macro programming language to simulate ametropia through the Macro language effectively constructed a customized optometry model. This enhanced the efficiency of the optical design and made possible the non-spherical optical design which was complex and time consuming. The optometry model in this paper can be used to determine the refraction results and used as a personal optometry model. These computational results with scientific evidence can be applied in future medical uses or customized 
TABLE 7. Recognition result of the network

\begin{tabular}{|c|c|c|c|c|c|c|c|c|c|c|c|c|c|c|c|c|c|c|c|c|c|c|c|c|c|}
\hline & 1 & 2 & 3 & 4 & 5 & 6 & 7 & 8 & 9 & 10 & 11 & 12 & 13 & 14 & 15 & 10 & 17 & 10 & 17 & 20 & & 22 & 23 & 24 & $\begin{array}{l}r \\
\text { on }\end{array}$ \\
\hline & & & & & & & ( & & 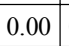 & & .00 & & 0 & & 0 & 0 & & 00 & & 0 & & 0 & & 0 & \\
\hline & & & & & & & & & & & & & & & & & & & & & & & & & \\
\hline & & & & & & & & & & & & & & & & & & & & & & & & & \\
\hline & & & & & & & & & & & & & & & & & & & & & & & & & \\
\hline & & & & & 8 & & ( & & $t$ & & 0.00 & & \begin{tabular}{|l|}
0.00 \\
\end{tabular} & & 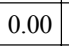 & 0 & ) & 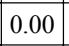 & & t & & & & 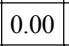 & \\
\hline & & & & & & & c & 0 & 0. & & 0. & & 0. & & 0.00 & 0 & 0 & 0 & & 0 & & 00 & 00 & 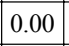 & \\
\hline & 0 & & & & ( & & 00 & & 0 & & 00 & & 0 & 0 & 0 & 0.00 & 0 & 00 & & 0 & & 0 & 00 & 0 & \\
\hline & & & & & & & & & 01 & & & & & & & & & & & & & & & & \\
\hline & & & & & & & & & & & & & & & & & & & & & & & & & \\
\hline & 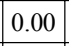 & & 0 & & c & & 0. & 0 & \begin{tabular}{|l|}
0.00 \\
\end{tabular} & 0 & \begin{tabular}{|l|}
0.00 \\
\end{tabular} & 0 & \begin{tabular}{|l|}
0.00 \\
\end{tabular} & 0 & \begin{tabular}{|l|}
0.00 \\
\end{tabular} & 0 & \begin{tabular}{|l|}
0.00 \\
\end{tabular} & 0. & 0 & 0 & ( & 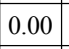 & 00 & ) & \\
\hline & & & & & 00 & & 0.00 & & \begin{tabular}{|l|}
0.00 \\
\end{tabular} & 0 & \begin{tabular}{|l|}
1.00 \\
\end{tabular} & & 00 & & 0 & 0.00 & 0 & 0. & & 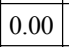 & & 00 & $x$ & 1 & \\
\hline & & & & & & & & & & & & & & & & & & & & & & & & & \\
\hline & & & & & & & & & & & & & & & & & & & & & & & & & \\
\hline & 1 & & & & 00 & 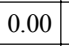 & \begin{tabular}{|l|l|}
0.00 \\
\end{tabular} & 0 & 0.00 & 0 & 0.00 & 0 & \begin{tabular}{|l|}
0.00 \\
\end{tabular} & 0 & \begin{tabular}{|l|}
0.00 \\
\end{tabular} & 0 & 0. & 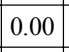 & ( & 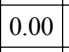 & & 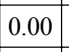 & 0.00 & 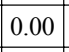 & \\
\hline & 00 & & & & \begin{tabular}{|l|}
0.00 \\
\end{tabular} & 0 & \begin{tabular}{|l|l|}
0.00 \\
\end{tabular} & 0.00 & \begin{tabular}{|l|l|}
0.00 \\
\end{tabular} & 0.00 & \begin{tabular}{|l|l|}
0.00 \\
\end{tabular} & 0 & \begin{tabular}{|l|}
0.00 \\
\end{tabular} & 1 & \begin{tabular}{|l|l|}
0.99 \\
\end{tabular} & 0.00 & \begin{tabular}{|l|}
0.00 \\
\end{tabular} & \begin{tabular}{|l|}
0.00 \\
\end{tabular} & 0 & \begin{tabular}{|l|}
0.00 \\
\end{tabular} & 00 & 0 & 0 & ) & \\
\hline & & & & & & & & & & & & & & & & & & & & & & & & & \\
\hline & & & & & & & & & & & & & & & & & & & & & & & & & \\
\hline & & & & & & & & & & & & & & & & & & & & & & & & & \\
\hline & 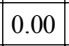 & & & & 00 & & 0.00 & 0 & 0.00 & 0 & 0.00 & 5 & 0 & ) & 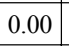 & 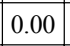 & 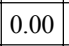 & 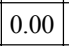 & 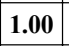 & 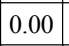 & & 0 & 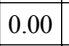 & 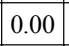 & 02 \\
\hline & & & & & & & & & 00 & & .00 & & & & & & & & & & & & 0 & & \\
\hline & 00 & & & & 0 & & ) & & 00 & & .00 & & 0 & 0 & 0 & 0 & 0 & 0 & & 2 & & 0.00 & 0 & 0 & 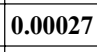 \\
\hline & & & & & 0.00 & & 0.00 & & 00 & & 00 & & 0 & & 0 & c & 00 & 0 & & 0 & & 0 & 00 & 0 & 00 \\
\hline $\mathrm{er} 23$ & $\rightarrow$ & & & & \begin{tabular}{|l|}
0.00 \\
\end{tabular} & & \begin{tabular}{|l|}
0.00 \\
\end{tabular} & \begin{tabular}{|l|}
0.00 \\
\end{tabular} & \begin{tabular}{|l|}
0.00 \\
\end{tabular} & & \begin{tabular}{|l|}
0.00 \\
\end{tabular} & & 0.00 & & 0 & 0 & 0.00 & 0.00 & 0 & \begin{tabular}{|l|}
0.00 \\
\end{tabular} & & 00 & 0 & 0 & - \\
\hline & & & & & & & & & 0.00 & & 0.00 & & & & & & & & & 0.00 & & 0.00 & & 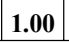 & \\
\hline
\end{tabular}

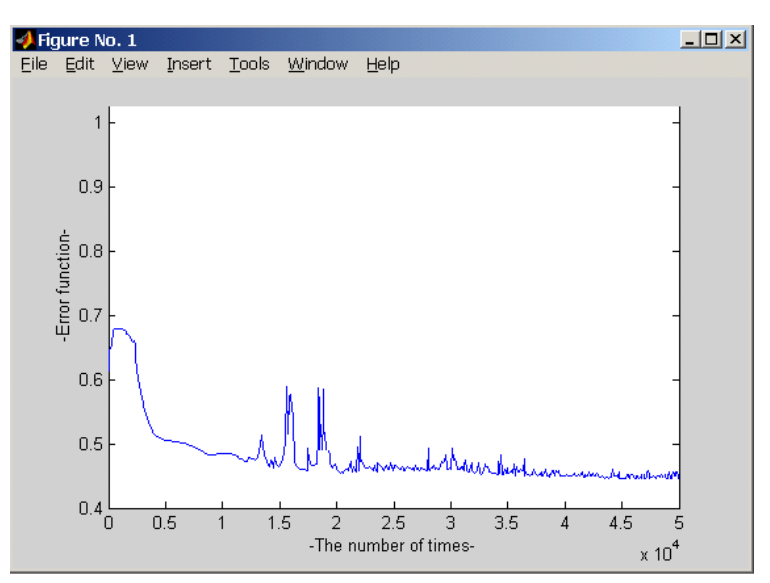

FIG. 5. Training curve with convergence of the network.

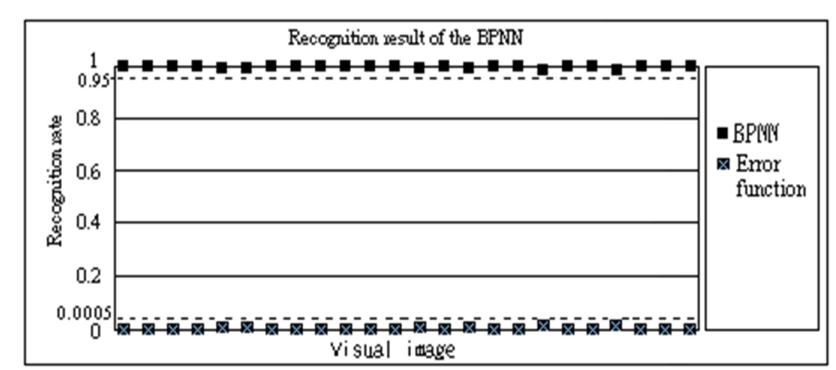

FIG. 6. Recognition result and error function. lenses. This paper focuses on the computation and recognition of myopia $(-0.25 \mathrm{D} \sim-6.00 \mathrm{D})$. Even though this approach already included the ametropia scope of most people, there is still room for improvement in the future, especially with regard to other customized designs with special needs and a high number of requirements.

\section{REFERENCES}

1. J. Cooper and K. Citek, "Comparison of refractive error measurements in adults with Z-view aberrometer, humphrey autorefractor, and subjective refraction," Optometry - Journal of the American Optometric Association 82, 231-240 (2011).

2. S. Prabakaran and M. Dirani, "Cycloplegic refraction in preschool children: comparisons between the hand-held autorefractor, table-mounted autorefractor and retinoscopy," Optik - International Journal for Light and Electron Optics, Ophthalmic Physiol. Optics 29, 422-426 (2009).

3. S. James, S. Paul, and B. Mark, "Evaluation of an automated subjective refractor," Optometry \& Vision Science 81, 334-340 (2004).

4. G. Smith, P. Bedggood, R. Ashman, M. Daaboul, and A. Metha, "Exploring ocular aberrations with a schematic human eye model," Optometry \& Vision Science 85, 330-340 (2008).

5. A. M. Storani and C. A. Carvalho, "Different schematic eyes and their accuracy to the in vivo eye: a quantitative 
comparison study," Journal of Physics 37, 378-387 (2007).

6. H. L. Liou and N. A. Brennan, "Anatomically accurate, finite model eye for optical modeling," J. Opt. Soc. Am. A 14, 1684-1695 (1997)

7. CODE V Reference Manual, "Introductory user's guide," Version 10.3, 14-143 (2011).
8. N. Lee, "Some applications of neural networks in optics and image processing," $\mathrm{Ph}$. D Thesis, University of Reading, J. J. Thomson Physics Lab, 48-71 (1998).

9. M. K. Hu, "Visual pattern recognition by moment invariants," IRE Transaction on Information Theory 8, 179-187 (1962).

10. Y. C. Fang and B. W. Wu, "Neural network application for thermal image recognition of low-resolution objects," Journal of Optics A: Pure and Applied Optics 9, 134-144 (2007). 